\title{
Hand Gesture Recognition Systems: A Survey
}

\author{
Arpita Ray Sarkar \\ National Institute of Tech., \\ Durgapur 713209, WB, India
}

\author{
G. Sanyal \\ National Institute of Tech., \\ Durgapur 713209, WB, India
}

\author{
S. Majumder \\ CSIR - CMERI, Durgapur \\ 713209, WB, India
}

\begin{abstract}
Gesture was the first mode of communication for the primitive cave men. Later on human civilization has developed the verbal communication very well. But still nonverbal communication has not lost its weightage. Such non verbal communication are being used not only for the physically challenged people, but also for different applications in diversified areas, such as aviation, surveying, music direction etc. It is the best method to interact with the computer without using other peripheral devices, such as keyboard, mouse. Researchers around the world are actively engaged in development of robust and efficient gesture recognition system, more specially, hand gesture recognition system for various applications. The major steps associated with the hand gesture recognition system are; data acquisition, gesture modeling, feature extraction and hand gesture recognition. There are several sub-steps and methodologies associated with the above steps. Different researchers have followed different algorithm or sometimes have devised their own algorithm. The current research work reviews the work carried out in last twenty years and a brief comparison has been performed to analyze the difficulties encountered by these systems, as well as the limitation. Finally the desired characteristics of a robust and efficient hand gesture recognition system have been described.
\end{abstract}

\section{General Terms}

Hand gesture recognition, comparison

\section{Keywords}

Data acquisition, gesture modeling, feature extraction, hand gesture recognition

\section{INTRODUCTION}

Gesture is a form of non-verbal communication using various body parts, mostly hand and face. Gesture is the oldest method of communication in human. Primitive men used to communicate the information of food/ prey for hunting, source of water, information about their enemy, request for help etc. within themselves through gestures. Still gestures are used widely for different applications on different domains. This includes human-robot interaction, sign language recognition, interactive games, vision-based augmented reality etc. Another major application of gestures is found in the aviation industry for placing the aircraft in the defined bay after landing, for making the passengers aware about the safety features by the airhostess. For communication by the people at a visible, but not audible distance (surveyors) and by the physically challenged people (mainly the deaf and dumb) gesture is the only method.

Posture is another term often confused with gesture. Posture refers to only a single image corresponding to a single command (such as stop), where as a sequence of postures is called gesture (such as move the screen to left or right). Sometimes they are also called static (posture) and dynamic gesture (gesture). Posture is simple and needs less computational power, but gesture (i.e. dynamic) is complex and suitable for real environments. Though sometimes face and other body parts are used along with single hand or double hands, hand gesture is most popular for different applications. A few of them are discussed below.

With the advancement of human civilization, the difficulty of interpersonal communication, not only in terms of language, but also in terms of communication between common people and hearing impaired people is gradually being abolished. If development of sign language is the first step, then development of hand recognition system using computer vision is the second step. Several works have been carried out worldwide using Artificial Intelligence for different sign languages.

Human-robot interaction [1] is another area where hand gesture recognition has been successfully used. The use of keyboard and mouse is limited to $2 \mathrm{D}$ world, but the controlling of a robot should be in 3D space. Hand gesture is most suitable for such purposes. However for robot control only a few simple commands are being used, such as the hand signal 'one' refers to 'move forward', 'five' refers to 'stop' and so on.

Similarly, for 3D CAD modeling inputs are provided by hand gestures. The 3-draw technology developed by MIT [2], is a pen embedded in polhemus device to track the position and orientation of the pen in 3D. A 3D space sensor is embedded in a flat palette that represents the plane of the object. The CAD model is moved synchronously with the user's gesture and objects can thus be rotated and translated in order to view them from all sides as they are being created and altered.

Other applications include Virtual Reality for communication media systems [3]; for controlling Television device to turn the TV on or off or changing the volume [4]; 3D gaming [5]. Different researchers are using different algorithms and features for the recognition. As mentioned, some of them are working in 2D and some of them are suitable for 3D environment. So these advancements in the field of hand gesture recognition need a complete review and also the different techniques used need to be analyzed.

The present work reviews a numbers of researches on hand gesture recognition systems along with the different steps of the recognition systems. A comparative study of all these works will provide the direction for work by the beginners as well as brief description of the steps associated with hand gesture recognition system. 


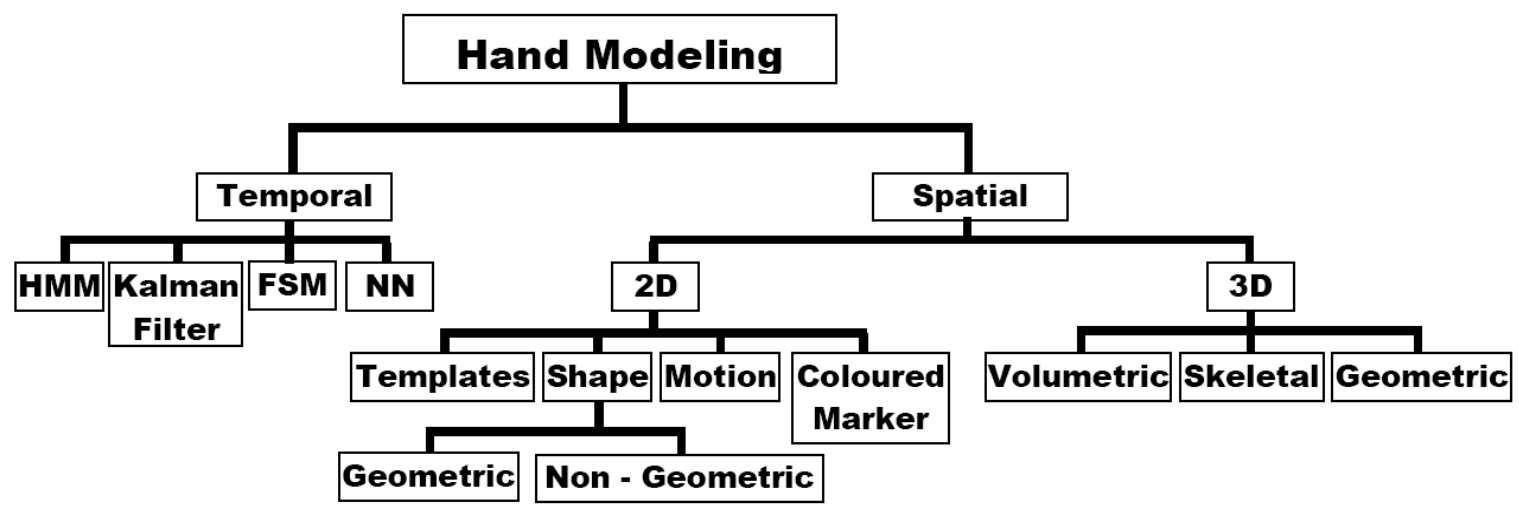

Fig 1: Classification of Hand Modeling [15]

\section{HAND MODELING FOR GESTURE RECOGNITION}

Human hand is an articulated object with 27 bones and 5 fingers [6][7]. Each of these fingers consists of three joints. The four fingers (little, ring, middle and index) are aligned together and connected to the wrist bones in one tie and at a distance there is the thumb. Thumb always stands on the other side of the four fingers for any operation, like capturing, grasping, holding etc. Human hand joints can be classified as flexion, twist, directive or spherical depending up on the type of movement or possible rotation axes. In total human hand has approximately 27 degrees of freedom. As a result, a large number of gestures can be generated.

Therefore, for proper recognition of the hand, it should be modeled in a manner understandable as an interface in Human Computer Interaction (HCI). There are two types of gestures [8], Temporal (dynamic) and Spatial (shape). Temporal models use Hidden Markov Model (HMM) [9], Kalman Filter [10], Finite State Machines [11], Neural Network (NN) [12][13]. Hand modeling in Spatial domain can be further divided into two categories, 2D (appearance based or view based) model and 3D based model [14][15].

2D hand modeling can be represented by deformable templates, shape representation features, motion and coloured markers [14]. Shape representation feature is classified as geometric features (i.e. live feature) and non-geometric feature [15]. Geometric feature deals with location and position of fingertips, location of palm and it can be processed separately. The non - geometric feature includes colour, silhouette and textures, contour, edges, image moments and Eigen vectors [14][16][17]. Non-geometric features cannot be seen (blind features) individually and collective processing is required [15]. The deformable templates are flexible in nature and allow changes in shape of the object up to certain limit for little variation in the hand shape. Image motion based model can be obtained with respect to colour cues to track the hand. Coloured markers are also used for tracking the hand and detecting the fingers/ fingertips to model the hand shape [18]. Hand shape can also be represented using 3D modeling. The hand shape in 3D can be volumetric, skeletal and geometric models. Volumetric models are complex in nature and difficult for computation in real-time applications. It uses a lot of parameters to represent the hand shape. In stead other geometric models, such as cylinders, ellipsoids and spheres are considered as alternative for such model for hand shape approximation [15]. Skeletal model represents the hand structure with 3D structure with reduced set of parameters. Geometric models are used for hand animation and real-time applications [18]. Polygon meshes and cardboard models are examples of geometric models. Figure 2 describes various hand modeling methods [15] to represent hand postures.

\section{SYSTEM ARCHITECTURE}

Hand gesture recognition system has four different phases to find out the gesture. They are data acquisition, hand segmentation and pre-processing, feature extraction and finally the recognition. The hand image is captured by suitable input device. The image is segmented to locate the hand from the (cluttered) background and other parts of the body and thereafter the image is processed to remove noises, to detect edges/ contours, to normalize for generating the simplest and desired model. The features are extracted from the segmented and pre-processed image for recognition. Finally the input images are recognized as a meaningful gesture based on the

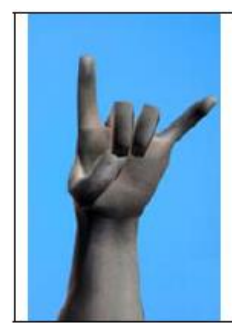

(a)

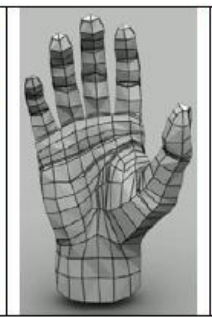

(b)

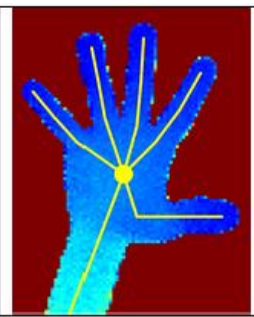

(c)

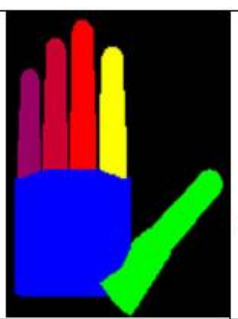

(d)

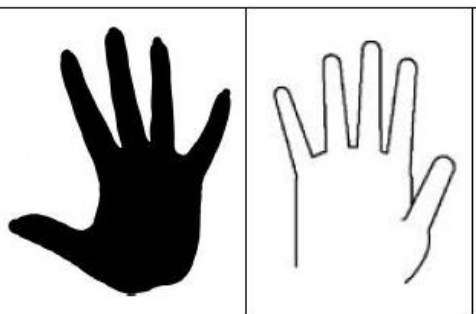

(e)

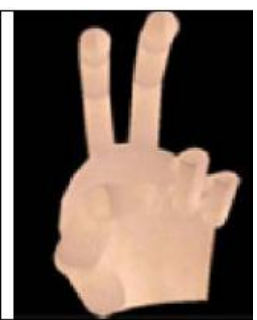

(g)

Fig - 2: Various hand modeling techniques [15] (a) 3D volumetric model (b) 3D geometric model (c) 3D skeleton model (d) coloured marker based model (e) Non - geometric shape model (Binary silhouette) (f) 2D deformable template model (contour) (g) Motion based model 
gesture modeling and analysis [14]. The details of the above phases are discussed in the following paragraphs. A schematic diagram of the popularly used hand gesture recognition system is shown in Figure 3 below.

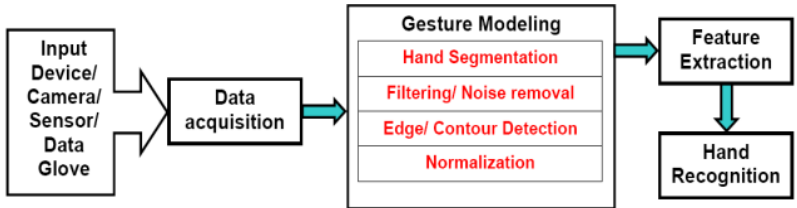

Fig 3: Generalized System Architecture for Hand Gesture Recognition

\subsection{Data Acquisition}

For efficient hand gesture recognition, data acquisition should be as much perfect as possible. Suitable input device should be selected for the data acquisition. There are a number of input devices for data acquisition. Some of them are data gloves, marker, hand images (from webcam/ stereo camera/ Kinect 3D sensor) and drawings [11][14][15][16][17][18][19]. Data gloves are the devices for perfect data input with high accuracy and high speed. It can provide accurate data of joint angle, rotation, location etc. for application in different virtual reality environments. At present, wireless data gloves are available commercially so as to remove the hindrance due to the cable. Coloured markers attached to the human skin are also used as input technique and hand localization is done by the colour localization. Input can also be fed to the system without any external costly hardware, except a low-cost web camera. Bare hand (either single or double) is used to generate the hand gesture and the camera captures the data easily and naturally (without any contact). Sometimes drawing models are used to input commands to the system. The latest addition to this list is Microsft Kinect 3D depth sensor [19]. Kinect is a 3D motion sensing input device widely used for gaming. It consists of a laser projector and a CMOS sensor for operation in any lighting conditions.

\subsection{Gesture Modeling}

It is the next step after data acquisition and the success of the gesture recognition mostly depends on this stage. Different data received through the input devices are to be modeled properly depending up on the type of applications. Gesture modeling has four different steps, [16][18] viz. hand segmentation, filter/ noise removal, edge/ contour detection and lastly normalization.

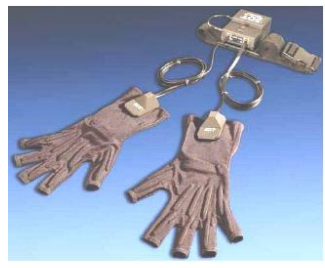

(a)

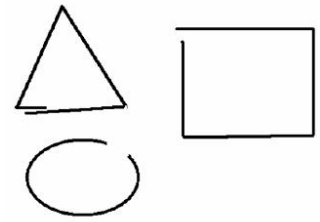

(d)

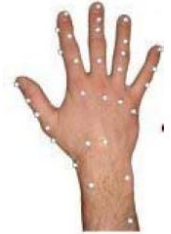

(b)

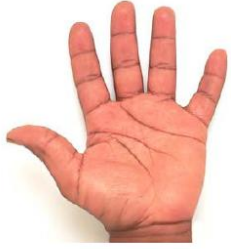

(c)

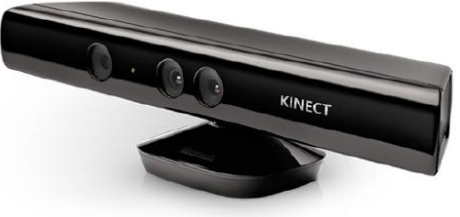

(e)
Fig 4: Different data input devices/methods (a) Wireless data gloves (available commercially) (b) Marker (c) Hand image (d) Drawings (e) Kinect 3D depth sensor

\subsubsection{Hand Segmentation}

Hand segmentation (or hand localization) refers to locate the hand or hand sequences in the images. There are different methods for hand segmentation and all of them classify the image in two homogeneous parts, foreground containing the hand and background containing the rest. Backgrounds are of two types, uniform [1][6][10]-[13][20][23]-[26][29][32][34][46][49]-[53][55][57]-[60][63][64][66][68][70]-[72][75][78][80][87] and cluttered [5][9][19][21][27][28][31][47][48][54] $[56][61][62][65][67][69][73][74][81][82][85][86]$. It is very necessary to locate the hand successfully within noisy/ cluttered background as uniform background is possible only in lab-scale trials. However background does not matter in case of data acquisition using contact type devices, such as key board-mouse, data gloves [83][84]etc. The following methods are most popular for hand segmentation.

\subsubsection{Thresholding}

In this method the image is divided in two regions of interest, background and foreground, based up on a particular value (termed as thresholding value). Thresholding is based on different real-time parameters, some of them use range/ depth thresholding [19][30][35][50], some of them use colour (RGB or HSV) thresholding [20][22][28][32][33], some use speed thresholding [76]. Otsu thresholding [55][81]is another important approach which performs histogram shape-based image thresholding or reduction of a grey level image to a binary image, assuming that the image contains only two types of pixels.

\subsubsection{Skin-based}

The human skin colour can be used to separate the hand, head or body from the back ground using RGB or Grey or HSV (hue, saturation and value) (also known as HSB i.e. hue, saturation and brightness) colour space representation [1][20][22][26][28][32][33][43] [46]. It can be used to separate the foreground from background with reference to predefined range of the colour.

\subsubsection{Subtraction}

In this approach the background of a test image can be separated with reference to a previously static image to find any changes in the test image [42][76]. This is very useful in finding moving objects in videos from static camera. A robust background subtraction method should be capable of handling lighting/ illumination change, repetitive motion from clutter and long-term scene changes [88].

\subsubsection{Statistical model}

The image is converted into a statistical model to assign a probabilistic value for each pixel to be a foreground and background loyalty. The probabilities will help to separate the foreground and background. Bayesian rule based [6], Gaussian mixture model [15][27][62], Expectation Maximization (EM) algorithm [77][82] are some of the common approaches being used presently.

\subsubsection{Colour normalization:}

The illumination is a factor that affects the distribution of color values in an image. These values change depending on different lighting conditions or different cameras. Colour normalization allows for object recognition techniques based on RGB colour, to compensate for these variations [83]. 


\subsubsection{Noise removal}

Noise is an important factor which affects the quality of an image. Noise is introduced either during the capturing or processing or transmission of the image. Noise removal or reduction is necessary for successful hand gesture recognition. Judicial selection of noise filter will lead to effective noise removal. There are many methods for noise removal.

\subsubsection{Salt and pepper}

It is the most common noise often found in an image. This appears as randomly occurring white and black pixels. This can be reduced by median filter, morphological filter, contra harmonic mean filter.

\subsubsection{Morphology erosion}

Morphological operations are mathematical operations designed in the context of set theory. Presently, this is used for noise reduction in image processing through two basic morphological operators [9][12][51][67][83], erosion and dilation. An erosion filter tends to reduce the sizes of bright image features by correlation with adjacent dark areas, where as the dilation filter does the opposite that means it constrains dark features with pixels from surrounding brighter areas. The opening of an image is defined as the erosion of the image followed by subsequent dilation using the same structural element.

\subsubsection{Multidimensional mean}

Here in stead of a single filter, a combination of 8 filters [15] is used to detect the edge noise or fluctuating for more edge smoothness.

\subsubsection{Edge detection}

Edge or contour detection is a technique which attempts to capture the significant properties of the image of the object [89]. Discontinuities in photometrical, geometrical and physical properties of the objects are contributing for such detection. These can be obtained by finding out the variations in terms of discontinuities (step edges), local extrema (line edges) and 2D features formed where at least two meet (junctions) in the grey level images. Edge detection helps to localize these variations and to search the reason behind. There are a number of edge detection techniques. They can be classified as [90] Gradient based Edge detection and Laplacian based Edge detection. The gradient method detects the edges by finding the maximum and minimum in the first derivative of the image, where as the Laplacian method determines the zero crossings in the second derivative of the image to detect edges. Some researchers have also classified the edge detectors as given below [15].

\subsubsection{First derivative}

The gradient method described above falls under this category. The edge is detected by the local maxima compared to its surrounding area with a higher value than a specific threshold.

\subsubsection{Second derivative}

If the contrast-change in not significant, edges can be detected using zero crossing. This is nothing but the Laplacian based edge detection. However, this may lead to generation of many false edges for which suitable and efficient pre-processing (blurring) operation should be executed.

\subsubsection{Morphological operations}

Some researchers have used this non-linear method [91] successfully for extraction of edge/ contour information in which erosion and image subtraction have been applied. However, the disadvantage of this method is that it is a time consuming technique.

The edge detection techniques mostly used [91] are; Sobel operator, Robert's cross operator, Prewitt's operator, Laplacian of Gaussian, Canny Edge Detection algorithm.

\subsubsection{Normalization}

The last step of gesture modeling is normalization or feature space reduction [15]. As the region of interest for any image is concentrated in a small area, therefore it is desirable to crop only the relevant area and then process it further. This will speed up the processing to locate the geometric features. Some of the operations used for this purpose are described below.

\subsubsection{Cropping operation}

The relevant area of the image containing the hand or face is cropped and fitted in a window removing the unnecessary background [21][45][53][68]. As a result the object will cover up the most of the image.

\subsubsection{Dimension unification}

This unification is necessary to set all the image sizes uniform to a specific dimension for better feature matching with the database. Different sizes have been used by different researchers depending up on the application, hardware etc., such as $320 \times 240$ by [68], $160 \times 120$ by [92].

\subsubsection{Significant features location}

The feature space can also be reduced for decrease in the computing time requirement. [93] has used Gabor filter to reduce the features from $6400(80 \times 80)$ to 35 . Some researchers have used different machine learning techniques for significant feature extraction. For example, neural network has been used by [94] for feature space reduction.

\subsection{Feature Extraction}

Features are the crucial elements for hand gesture recognition. Large number of features, such as, shape, orientation, textures, contour, motion, distance, centre of gravity etc. can be used for hand gesture recognition. Hand gesture can be recognized using geometric features, like, hand contour, fingertips, finger detections. But these features may neither be always available nor reliable due to occlusions and illuminations [16]. Some non-geometric features (such as colour, silhouette, texture) are also available for recognition. But they are inadequate for the purpose. Therefore, the image or the processed image can be fed to the recognizer to select the features automatically and implicitly, rather than using single type of feature alone. Following three approaches [16] are useful for extraction of features.

\subsubsection{Model based (Kinematic model) approach}

The palm pose and joint angles have been used as features in [10][20][25]. This method is suitable in case of real-time interaction in virtual environment. Here, those kinematic parameters, which bring the $2 \mathrm{D}$ projection of a $3 \mathrm{D}$ hand model in correspondence, are searched. But the difficulty with this approach [16] lies in the process of feature (i.e. edge) extraction, because human hands are texture less and do not provide reliable edges internally. For reliable and effective 
extraction of model based features, homogeneous and high contrast background with reference to the hand is essential.

\subsubsection{View based approach}

The difficulties of model based approach can be overcome by using view based approach [16]. In this method, the hand is modeled by a collection of 2D intensity images.

\subsubsection{Low-level-features based approach}

For gesture recognition it is necessary to map the input video or the sequences from the video to the predefined gesture from the database. But reconstruction of the complete hand model is not essential argued by some researchers [16]. However, several low-level image measurements those are relatively robust to noise and can be extracted quickly have been adapted. Some of the low-level features used for gesture recognition are: centroid of hand [6][30], optical flow and principal axes defining an elliptical bounding region of the hand [16].

\subsection{Hand Gesture Recognition}

Once the appropriate features as mentioned above, have been extracted from the images and a suitable data set have been selected, the gestures can be recognized using standard machine learning techniques or a special-purpose classifiers. Several methods have been used for gesture recognition: template matching, dictionary look-up, statistical matching, linguistic matching, neural network and ad hoc method.

Hidden Markov Model (HMM) is a doubly stochastic model and appropriate for dealing with the stochastic properties in gesture recognition. As a first choice, researchers prefer to use Hidden Markov Model (HMM) [9][10][35][65][67][73][78] [81][84][85] for the data containing temporal information for dynamic hand gesture recognition. Another advantage of HMM is its high recognition rates. Some researchers also have used HMM combining with other classifiers. [41] has used dynamic Bayesian network, conditional random fields (CRF) is used in [62]. Also the K-nearest neighbour and Support Vector Machine (SVM), has been fused with HMM [58] for faster recognition in spite of its simplicity.

Takagi - Sugeno - Kang (TSK) or simply Sugeno type fuzzy inference system has been used in [31] [36]. The main operation of Sugeno-type fuzzy inference system is by the generation of fuzzy rules from input -output data set. Neural network [13][27][66] is another widely used classifier which uses predefined database for correct matching. Different forms of neural network have been widely used by different researchers. Some of them are, K-mean based radial basis function neural network [12], feed-forward back propagation neural network [29], back propagation neural network [42] where the network learns from the desired output. Multi layer Perceptron (MLP) is another method of neural network which has been successfully used in [63][70][74][79]. This class of networks consists of multiple layers of computational units, usually interconnected in a feed-forward way.

Another method popularly used is Support Vector Machine (SVM). SVM is a supervised learning and takes a set of input data and predicts the output, for each given input based on its training on standard available data set. For proper utilization of SVM classifier, one needs to know four basic concepts: separating hyperplane, maximum-margin hyperplane, soft margin and the kernel function. In some cases only basic
SVM have been used [23][37][46]. Some researches used it in other form, such as Multiclass SVM [48][59][61] or in a fused form [51][58][59] with other methods.

Kalman filter, or linear quadratic estimation (LQE), is an estimation method that utilizes a series of measurements observed over time, containing noise and other inaccuracies, and generates estimates of unknown variables that tend to be more precise. Methods derived using Kalman filter, such as coupled - forward algorithm [77] or fused with Kalman filter, such as Kalman filter and collapsing method [82], have been used for successful recognition.

Some researchers have also used [34][54] Principal Component Analysis (PCA) for successful recognition of gestures. It uses an orthogonal transformation to convert a set of observations of possibly correlated variables into a set of values of linearly uncorrelated variables called principal components.

Haar-like techniques use Haar wavelet for successful gesture recognition [22][26]. This approach considers adjacent rectangular regions at a specific location in a detection window and then adds up the pixel intensities in each region and calculates the difference between these sums.

Apart from the above, other classifier, such as Correlation [21], normalized correlation [5], minimum distance classifier [6][52][60], Probabilistic model [25], Iterative Closest Point (ICP) matching - voxel quantization - Binary matching [28], Heuristic and voxel-based algorithm [30], Genetic algorithm [33], Action graph [38], contour matching [43], Term Frequency - Inverse Document Frequency [44], Polygon matching algorithm [47], Template matching [50], Particle swarm optimization [55], Geometrical indexing [57], Dynamic Time Wrapping [59], boundary tracing [64], predictive eigen tracker [72], Hausdorff matching [75], Particle filtering [76], silhouette modeling [86] have been used in case-to-case basis by different researchers successfully.

It is evident that some of the above methods are supervised in nature and some are unsupervised. Supervised classifiers have used already available and proven database for gesture recognition. In some cases, where such database is not available, unsupervised method has been used.

\section{4. $\quad$ Research Results}

Hand gesture recognition is a challenging interdisciplinary domain which uses computer vision and graphics, image processing, machine learning techniques, bio-informatics and psychology [16] for successful operation. The overall research summary is presented in two tables as below. Table 1 describes the type of backgrounds used, methods adapted for segmentation, extracted features and the recognition techniques along with accuracies. 
Table 1. Comparison of different parameters of surveyed HGR systems

\begin{tabular}{|c|c|c|c|c|c|}
\hline $\begin{array}{l}\text { Method } \\
\text { [Ref. No.] }\end{array}$ & Background & $\begin{array}{l}\text { Segmentation } \\
\text { technique }\end{array}$ & $\begin{array}{l}\text { Feature Vector } \\
\text { Representation }\end{array}$ & Classifier/ Recognition & $\begin{array}{l}\text { Accuracy } \\
(\%)\end{array}$ \\
\hline$[1]$ & uniform & $\begin{array}{l}\text { Red Green ratio of } \\
\text { the skin colour }\end{array}$ & $\begin{array}{l}\text { Centre of gravity and } \\
\text { farthest distance }\end{array}$ & - & 91 \\
\hline [5] & cluttered & Thresholding & $\begin{array}{c}\text { Orientation } \\
\text { representation }\end{array}$ & Normalized correlation & - \\
\hline [6] & Uniform & $\begin{array}{l}\text { Bayesian rule based } \\
\text { skin colour }\end{array}$ & $\begin{array}{l}\text { Centroid, Normalization } \\
\text { constant \& orientation }\end{array}$ & Minimum distance & 93.37 \\
\hline [9] & Cluttered & Thresholding & $\begin{array}{l}\text { Hand position, velocity, } \\
\text { size and shape }\end{array}$ & HMM & 96.67 \\
\hline$[10]$ & Uniform & $\begin{array}{c}\text { Infrared } \\
\text { Thresholding } \\
\end{array}$ & Centre of palm & HMM & $\begin{array}{l}\text { Single } 99.2 \\
\text { Double } 97.5 \\
\end{array}$ \\
\hline [12] & Uniform & $\begin{array}{l}\text { Histogram based } \\
\text { Thresholding }\end{array}$ & $\begin{array}{l}\text { Localized Contour } \\
\text { Sequence }\end{array}$ & $\begin{array}{c}\text { K-mean based Radial } \\
\text { Basis Function Neural } \\
\text { Network }\end{array}$ & 99.6 \\
\hline [13] & uniform & $\begin{array}{l}\text { Orientation } \\
\text { histogram }\end{array}$ & Euclidean distance & Neural Network & - \\
\hline [19] & Clutter & Depth Thresholding & Convex hull and contour & $\begin{array}{c}\text { Finger counting, Finger } \\
\text { name collecting \& } \\
\text { Vector matching } \\
\text { classifier }\end{array}$ & $\begin{array}{l}\text { Single } 84 \\
\text { Double } 90\end{array}$ \\
\hline$[20]$ & uniform & Colour based HSV & $\begin{array}{l}\text { Euclidean distance from } \\
\text { the boundary, centre of } \\
\text { palm }\end{array}$ & - & - \\
\hline$[21]$ & Cluttered & Thresholding & $\begin{array}{l}\text { Scale invariant features } \\
\text { computed at the edge }\end{array}$ & Correlation & - \\
\hline [22] & uniform & HSV colour space & $\begin{array}{l}\text { Convex hull of the } \\
\text { contour }\end{array}$ & Haar like technique & - \\
\hline [23] & Uniform & Thresholding & Boundary, convex hull & $\begin{array}{c}\text { Support Vector } \\
\text { Machine }\end{array}$ & 80 \\
\hline$[25]$ & Uniform & Thresholding & $\begin{array}{l}\text { Mean Euclidean distance, } \\
\text { mean turning angle }\end{array}$ & Probabilistic model & $\begin{array}{l}\text { Single } 92.7 \\
\text { Double } 96.2\end{array}$ \\
\hline$[26]$ & Uniform & HSV colour space & $\begin{array}{l}\text { Contour, convex hull of } \\
\text { the contour }\end{array}$ & Haar like technique & - \\
\hline [27] & Cluttered & Active contour & Colour, shape and texture & Neural network & 93 \\
\hline [28] & Cluttered & RGB of skin colour & Angular pose & $\begin{array}{l}\text { Iterative Closest Point } \\
\text { (ICP) matching, Voxel } \\
\text { quantization and binary } \\
\text { matching }\end{array}$ & - \\
\hline [29] & $\begin{array}{l}\text { Dark and } \\
\text { uniform }\end{array}$ & Thresholding & Contour & $\begin{array}{c}\text { Feed Forward Back } \\
\text { Propagation Neural } \\
\text { Network }\end{array}$ & 95.34 \\
\hline [30] & $\begin{array}{l}\text { No special } \\
\text { background }\end{array}$ & Range thresholding & Centroid of hand & $\begin{array}{l}\text { Heuristic and voxel- } \\
\text { based algorithm }\end{array}$ & 98.24 \\
\hline$[31]$ & Cluttered & $\begin{array}{l}\text { Energy minimization } \\
\text { of active contours }\end{array}$ & $\begin{array}{l}\text { Colour, texture, boundary } \\
\text { edge map and prior shape } \\
\text { information }\end{array}$ & $\begin{array}{l}\text { Takagi-sugeno-kang or } \\
\text { Sugeno type Fuzzy } \\
\text { inference system }\end{array}$ & 96 \\
\hline$[32]$ & Uniform & HSV colour space & $\begin{array}{l}\text { Centre of hand and palm } \\
\text { size (radius) }\end{array}$ & Simple matching & - \\
\hline [33] & - & HSV colour model & $\begin{array}{l}\text { Hausdoff distance and } \\
\text { Fourier descriptor }\end{array}$ & Genetic algorithm & - \\
\hline [34] & Uniform & $\begin{array}{l}\text { Pixel intensity } \\
\text { thresholding }\end{array}$ & Haar-like features & $\begin{array}{l}\text { Principal Component } \\
\text { Analysis (PCA) }\end{array}$ & $\begin{array}{c}\text { Hand }-94.63 \\
\text { Face }-98.4 \\
\end{array}$ \\
\hline$[35]$ & $\begin{array}{l}\text { Mostly } \\
\text { uniform }\end{array}$ & Depth thresholding & Sparse depth map & HMM & 94 \\
\hline$[36]$ & Uniform & Thresholding & Fourier descriptor & $\begin{array}{l}\text { Sugeno type Fuzzy } \\
\text { inference system }\end{array}$ & 96 \\
\hline$[37]$ & Uniform & $\begin{array}{l}\text { Maximum Curvature } \\
\text { Point (MCP) }\end{array}$ & Location of Key MCPs & SVM & $\begin{array}{c}\text { Numerals } \\
93.2\end{array}$ \\
\hline [38] & Uniform & Depth Thresholding & Cell occupancy and & Action graph & 87.7 \\
\hline
\end{tabular}




\begin{tabular}{|c|c|c|c|c|c|}
\hline & & & silhouette & & \\
\hline [41] & Uniform & $\begin{array}{c}\text { Colour-based region- } \\
\text { of-interest }\end{array}$ & Active contour & $\begin{array}{c}\text { Bi-driven inference } \\
\text { (Dynamic Bayesian } \\
\text { Network + HMM) } \\
\end{array}$ & 95 \\
\hline [42] & Uniform & $\begin{array}{l}\text { Background } \\
\text { subtraction with } \\
\text { reference image }\end{array}$ & $\begin{array}{l}\text { Wavelet network } \\
\text { parameters }\end{array}$ & $\begin{array}{l}\text { Back propagation } \\
\text { Neural Network }\end{array}$ & 97 \\
\hline [43] & $\begin{array}{l}\text { Mostly } \\
\text { uniform }\end{array}$ & $\mathrm{YCrCb}$ colour space & HU moment & Contour matching & 89.53 \\
\hline [44] & $\begin{array}{l}\text { Mostly } \\
\text { uniform }\end{array}$ & $\begin{array}{l}\text { Divergence field of } \\
\text { optical flow }\end{array}$ & $\begin{array}{l}\text { Maximally Stable } \\
\text { External Region }\end{array}$ & $\begin{array}{l}\text { Term Frequency - } \\
\text { Inverse Document } \\
\text { Frequency } \\
\end{array}$ & 97.62 \\
\hline [45] & Uniform & $\mathrm{YCrCb}$ colour space & $\begin{array}{l}\text { Centroid, thumb and } \\
\text { finger region detection, } \\
\text { Euclidean distance }\end{array}$ & Thresholding & 94 \\
\hline [46] & Uniform & HSV colour space & $\begin{array}{c}\text { Euclidean distance } \\
\text { between centroid of hand } \\
\text { and fingertip }\end{array}$ & SVM & - \\
\hline [47] & Cluttered & $\begin{array}{l}\text { HSV colour space, } \\
\text { Depth Thresholding }\end{array}$ & $\begin{array}{l}\text { HU moment (HUM) and } \\
\text { Turning-angle distance } \\
\text { (TD) }\end{array}$ & $\begin{array}{l}\text { Polygon matching } \\
\text { algorithm }\end{array}$ & $\begin{array}{c}\text { TD - } 85 \\
\text { HUM - } 58\end{array}$ \\
\hline [48] & Cluttered & HSV colour space & Haar-like features & Multiclass SVM & 96.23 \\
\hline [49] & Uniform & $\mathrm{YCrCb}$ colour space & $\begin{array}{l}\text { Centroid, thumb and } \\
\text { finger region detection, } \\
\text { Euclidean distance }\end{array}$ & Thresholding & 92 \\
\hline [50] & Uniform & Depth Thresholding & $\begin{array}{c}\text { Finger-Earth Mover's } \\
\text { Distance } \\
\end{array}$ & Template matching & 92.25 \\
\hline [51] & Uniform & Otsu thresholding & Local contour sequence & $\begin{array}{c}\text { SVM and Least-square } \\
\text { SVM }\end{array}$ & $\begin{array}{c}\text { SVM - } 98.6 \\
\text { LSSVM - } \\
99.2\end{array}$ \\
\hline [52] & $\begin{array}{l}\text { Mostly } \\
\text { uniform }\end{array}$ & $\begin{array}{c}\text { Bayesian rule based } \\
\text { RGB colour }\end{array}$ & $\begin{array}{l}\text { Euclidean distance and } \\
\text { angles }\end{array}$ & Distance classifier & 93.38 \\
\hline [53] & Uniform & HSV colour space & $\begin{array}{l}\text { Weighted value of } \\
\text { parameters of the } \\
\text { curve/line equation }\end{array}$ & $\begin{array}{l}\text { Real time Image } \\
\text { Comparison } \\
\text { (online)/Attributes } \\
\text { Matching (offline) }\end{array}$ & - \\
\hline [54] & Cluttered & HSV colour space & contour & PCA & 92.95 \\
\hline [55] & Uniform & $\begin{array}{l}\text { Otsu thresholding } \\
\text { (colour and depth) }\end{array}$ & Hu moments & $\begin{array}{c}\text { Particle Swarm } \\
\text { Optimisation }\end{array}$ & - \\
\hline [56] & Cluttered & RGB colour space & $\begin{array}{l}\text { Euclidean distance and } \\
\text { angle }\end{array}$ & Simple comparison & 94.21 \\
\hline [57] & $\begin{array}{c}\text { Mostly } \\
\text { uniform }\end{array}$ & CIELab colour space & $\begin{array}{c}\text { Geometric feature of } \\
\text { convex hull of skin blobs }\end{array}$ & Geometrical indexing & 92.34 \\
\hline [58] & Uniform & $\begin{array}{l}\text { RGB colour } \\
\text { thresholding }\end{array}$ & $\begin{array}{c}\text { Speed Up Robust } \\
\text { Features (SURF) and Hu } \\
\text { moments }\end{array}$ & $\begin{array}{l}\text { K-Nearest Neighbour } \\
\text { and SVM/ HMM }\end{array}$ & Word - 96 \\
\hline [59] & Uniform & $\mathrm{YCrCb}$ colour space & $\begin{array}{l}\text { Shape, texture and finger } \\
\text { features }\end{array}$ & $\begin{array}{l}\text { Multiclass non-linear } \\
\text { SVM (static), DTW } \\
\text { (dynamic) }\end{array}$ & $\begin{array}{c}\text { Static }-91.3 \\
\text { Dynamic - } \\
86.3\end{array}$ \\
\hline [60] & Uniform & $\begin{array}{l}\text { YCrCb and YIQ } \\
\text { colour space }\end{array}$ & $\begin{array}{c}\text { Geometric and } \\
\text { orthogonal (Zernike }(\mathrm{Z}), \\
\text { Tchebichef }(\mathrm{T}) \& \\
\text { Krawtchouk }(\mathrm{K})) \\
\text { moments } \\
\end{array}$ & $\begin{array}{l}\text { Minimum distance } \\
\text { classifer }\end{array}$ & $\begin{array}{c}\text { Geometric } \\
88.2 \\
\mathrm{Z}-94.5 \\
\mathrm{~T}-97.7 \\
\mathrm{~K}-98.4 \\
\end{array}$ \\
\hline [61] & Cluttered & - & SIFT features & Multiclass SVM & 96.25 \\
\hline [62] & Fully cluttered & $\begin{array}{l}\text { YCrCb colour space } \\
\text { and depth }\end{array}$ & $\begin{array}{c}\text { State and transition } \\
\text { feature }\end{array}$ & $\begin{array}{c}\text { HMM, Conditional } \\
\text { Random Fields (CRF) }\end{array}$ & $\begin{array}{c}\text { HMM - } 93.31 \\
\text { CRF }-90.49 \\
\end{array}$ \\
\hline [63] & $\begin{array}{l}\text { Mostly } \\
\text { uniform }\end{array}$ & $\mathrm{YCrCb}$ colour space & $\begin{array}{l}\text { Hu variant moment, hand } \\
\text { gesture region and } \\
\text { Fourier descriptor }\end{array}$ & $\begin{array}{l}\text { Multi Layer Perceptron } \\
\text { (MLP) }\end{array}$ & 97.4 \\
\hline [64] & Uniform & Canny edge detector & Texture and shape & Boundary tracing & 95 \\
\hline [65] & Fully cluttered & $\begin{array}{l}\text { YCrCb colour space } \\
\text { and 3D depth map }\end{array}$ & $\begin{array}{l}\text { Location, orientation and } \\
\text { velocity }\end{array}$ & HMM & $\begin{array}{c}\text { Isolated } 98.94 \\
\text { Cont. } 95.7 \\
\end{array}$ \\
\hline [66] & Uniform & Thresholding & Area & NN & 92.07 \\
\hline [67] & Fully cluttered & YCrCb colour space & Location, orientation and & HMM & 92.3 \\
\hline
\end{tabular}




\begin{tabular}{|c|c|c|c|c|c|}
\hline & & and 3D depth map & velocity & & \\
\hline [68] & $\begin{array}{l}\text { Uniform (light } \\
\text { and then dark) }\end{array}$ & - & Blob and ridge of hand & $\begin{array}{c}\text { Comparison of } \\
\text { geometric configuration }\end{array}$ & 83.85 \\
\hline [70] & Uniform & HSV colour space & Hand blob & MLP & 92 \\
\hline [72] & Uniform & 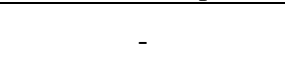 & Mahalanobis distance & $\begin{array}{c}\text { Predictive Eigen } \\
\text { Tracker }\end{array}$ & 100 \\
\hline [73] & Cluttered & Thresholding & Fourier descriptor & HMM & 90 \\
\hline [74] & Cluttered & Skin colour filter & LCS features & MLP & 84.8 \\
\hline [75] & Uniform & HIS colour space & $\begin{array}{l}\text { Bi-directional partial } \\
\text { Hausdorff distance }\end{array}$ & $\begin{array}{l}\text { Hausdorff matching } \\
\text { approach }\end{array}$ & 90 \\
\hline [76] & Cluttered & $\begin{array}{l}\text { Manually from the } \\
\text { background }\end{array}$ & Blob and ridge features & Particle filtering & 86.5 \\
\hline [77] & Cluttered & Skin colour model & Active contour & $\begin{array}{l}\text { Coupled-forward } \\
\text { algorithm based on } \\
\text { Kalman filter } \\
\end{array}$ & - \\
\hline [78] & Uniform & Speed thresholding & Change of direction & HMM & 94 \\
\hline [79] & Uniform & RGB colour space & Boundary chord's size & MLP & 98.7 \\
\hline [80] & Uniform & Skin-tone blob & $\begin{array}{l}\text { End point of finger using } \\
\text { width and height }\end{array}$ & Own method (SAVI) & 95.4 \\
\hline [81] & Cluttered & $\begin{array}{l}\text { Otsu Thresholding of } \\
\text { skin - colour }\end{array}$ & $\begin{array}{l}\text { Location, orientation and } \\
\text { velocity of centroid of the } \\
\text { hand }\end{array}$ & HMM & 85 \\
\hline [82] & Cluttered & Skin colour model & Active contour & $\begin{array}{c}\text { Forward algorithm } \\
\text { based on Kalman filter } \\
\text { and collapsing method }\end{array}$ & - \\
\hline [83] & Data gloves & $\begin{array}{c}\text { Normalized RGB } \\
\text { space colour }\end{array}$ & $\begin{array}{c}\text { 20-dimensional feature } \\
\text { vector }\end{array}$ & $\begin{array}{c}\text { Improved CombNET - } \\
\text { II (NN) }\end{array}$ & 99.4 \\
\hline [84] & Data Glove & $\begin{array}{l}\text { Discontinuity (time- } \\
\text { variant-parameter) } \\
\text { detection }\end{array}$ & $\begin{array}{l}\text { Posture, position, } \\
\text { orientation and motion }\end{array}$ & HMM & 80.4 \\
\hline [85] & Cluttered & Time duration & $\begin{array}{c}\text { 8-D chain code (each } \\
\text { hand's } x \text { and y position, } \\
\text { angle of axis of least } \\
\text { inertia and eccentricity of } \\
\text { bounding ellipse }\end{array}$ & HMM & 95.97 \\
\hline [86] & Cluttered & Thresholding & Disparity & $\begin{array}{c}\text { Multiple silhouette } \\
\text { models }\end{array}$ & - \\
\hline [87] & Uniform & Thresholding & $\begin{array}{l}\text { C.o.G, Finger tip position } \\
\text { and direction }\end{array}$ & $\begin{array}{l}\text { Ghost correspondence } \\
\text { eliminating table with } \\
\text { 3D prediction model }\end{array}$ & - \\
\hline
\end{tabular}

The maximum range of accuracy of the reviewed HGR systems lies between $90 \%$ and $100 \%$. [72] has obtained 100\% accuracy using Mahalanobis distance as feature and Predictive Eigen tracker as classifier/ recognizer.

There are several limitations of the above systems. They can be formulated as below.

Change in illumination: When there is a change in the illumination condition, the system fails to recognize properly [6][9][12][23][[28][29][33][[41]. Even if there is variation in lighting condition between the training dataset and inputs, some system fails to recognize.

Difficult background: About more than $60 \%$ of the papers use uniform background [1][6][10][12][13][20][22]-[26][32][46][49]-[53][55][57]-[60] for their recognition. But in real time gesture recognition uniform background is not desirable or available.

Rotation or orientation limitation: An HGR system fails to recognize if the hand is orientated in a different angle $[5][10][13][[19][22]-[29]$ with reverence to that in database.

Scaling problem: The problems of scaling arise due to different field of applications, hand size of the users, perspective.
Translation problem: The variation of hand positions [23][29][31]-[47][49]-[57] in different images also leads to erroneous representation of features.

Skin-like-coloured objects: Sometimes objects with similar colour that of human skin may be present in the environment and this leads to confusion of [1]-[18][20][29][32]-[87] the recognition systems.

Special hardware: A number of special hardware, like Range camera [30], 3D depth sensor [35][[38], Data gloves [83][84] have been used.

Each and every model has certain limitation. Some researchers have addressed one issue, some have others. There is not a single work that addresses all the issues simultaneously. From the above discussions and broad literature review, it has been found that four criteria are necessary for the best hand gesture recognition.

\section{Robustness:}

There several factors [16], such as, change in illuminations, clutter and dynamic backgrounds, occlusions, image resolution, wrong orientation, different user etc. which affect the image quality making it difficult for recognition. These factors make the image very rich, noisy or incomplete. The recognition system should be able to easily adopt these 
changes for successful recognition. Simultaneously the system should be robust enough to overcome the user dependency, colour (of background or foreground) dependency, device dependency etc.

\section{Computational efficiency:}

As most of the hand gesture recognition systems use different segmentation techniques, preprocessing, feature extraction and finally simple/ statistical matching or machine learning techniques, using mathematical approach, they need huge computation power as well as processing time. For an efficient hand gesture recognition system, these factors should be optimum and as a result cost efficient.

\section{User's tolerance:}

Human errors are very common in nature. HGR systems should easily accommodate such mistakes or malfunction of the systems without making wrong decision. Rather it should ask the signer the repeat the gesture.

\section{Scalability:}

Gestures in different scales based upon the application should be easily accommodated by the HGR systems. The same system should be useful for virtual reality application, 3D gaming, sign language interpretation, robot navigation, TV control etc. without any change, irrespective of the hardware.

\section{CONCLUSION}

Hand gesture recognition is finding its application for nonverbal communication between human and computer, general fit person and physically challenged people, 3D gaming, virtual reality etc. With the increase in applications, the gesture recognition system demands lots of research in different directions. A large number of research works carried out during last twenty years have been reviewed. The different sub-components, methodologies used for recognition of mainly hand gestures in those works have been described. A brief comparison of backgrounds, segmentation techniques, features used and the recognition methods have been done and presented. Finally the drawbacks and requirements for a perfect hand gesture recognition system have been discussed.

\section{ACKNOWLEDGMENTS}

Authors are also grateful to Dr. D. N. Ray for his continuous suggestions and advices, without which this work would not have completed.

\section{REFERENCES}

[1] Malima, A., Ozgur, E. and Cetin, M. 2006. A Fast Algorithm for Vision-Based Hand Gesture Recognition for Robot Control. In Proc. of 14th IEEE Signal Processing and Communications Applications

[2] Sachs, E. 1989. 3-Draw: a three dimensional computer aided design tool. In Proc. of IEEE International Conference on Systems, Man and Cybernetics

[3] Hyunjin, A., Daijin, K. 2009. Hand Gesture Recognition using 3D Depth Data. Dept. of CSE. Pohang University of Science and Technology, Korea

[4] LaViola Jr., J. J. 1999. A Survey of Hand Posture and Gesture Recognition Techniques and Technology. Masters' Thesis, Science and Technology Center for Computer Graphics and Scientific Visualization, USA.
[5] Freeman, W. T., Weissman, C. D. (1995). Television Control by Hand Gestures. IEEE International Workshop on Automatic Face and Gesture Recognition

[6] Bhuyan, M. K., Neog, D. R. and Kar, M. K. Fingertip Detection for Hand Pose Recognition. Int. J. On Computer Sc. and Engg. , 4(3) (March 2012), 501-511

[7] Moeslund, T. B., Hilton, A. and Kruger, V. A Survey of Advances in Vision-Based Human Motion Capture and Analysis. Computer Vision and Image Understanding, 104(2) (2006), 90 - 126

[8] Garg, P., Aggarwal, N. and Sofat, S. Vision Based Hand Gesture Recognition. World Academy of Science, Engineering and Technology, 49 (2009), 972- 977

[9] Yang, Z., Li, Y., Chen, W. and Zheng, Y. (2012) Dynamic Hand Gesture Recognition Using Hidden Markov Models. In Proc. of 7th Int. Conf. on Comp. Sc. and Education

[10] Oka, K., Sato, Y. and Koike, H. (2002) Real-time Tracking of Multiple Fingertips and Gesture Recognition for Augmented Desk Interface Systems. 5th IEEE Int. Conf. on Automatic Face and Gesture Recognition

[11] Wu, Y. and Huang, T. S. (1999) Vision-Based Gesture Recognition: A Review. Int. Gesture Workshop on Gesture-Based Communication in Human-Computer Interaction. Springer Lecture Notes in Computer Science

[12] Ghosh, D. K. and Ari, S. (2011) A Static Hand Gesture Recognition Algorithm using K-Mean based Radial Basis Function Neural Network. In Proc. of 8th Int. Conf. on Information, Communications \& Signal Processing

[13] Symeonidis, K. 2000. Hand Gesture Recognition Using Neural Networks. MS Thesis. University of Surrey, UK

[14] Pavlovic, V. I., Sharma, R. and Huang, T. S. Visual Interpretation of Hand Gestures for Human - Computer Interaction: A Review. IEEE Transactions on Pattern Analysis And Machine Intelligence, 19(7) (1997), 677 695

[15] Hasan, M. M. and Mishra, P. K. Hand Gesture Modeling and Recognition using Geometric Features: A Review. Canadian J. on Image Processing and Computer Vision, 3(1)1 (2012)

[16] Murthy, G. R. S. and Jadon, R. S. A Review of Vision Based Hand Gestures Recognition. Int. J. of Information Technology and Knowledge Management, 2(2) (2009), $405-410$

[17] Erol, A., Bebis, G., Nicolescu, M., Boyle, R. D., and Twombly, X. Vision Based Hand Pose Estimation: A Review. Computer Vision and Image Understanding, 108 (2007), 52-73

[18] Wu, Y. and Huang, T. S. Hand Modeling, analysis and Recognition. IEEE Signal Processing Magazine, 18(3) (2001), 51 - 60

[19] Li, Y. 2012. Hand Gesture Recognition using KINECT. MS thesis. University of Louisville

[20] Rajesh, J. R., Nagarjunan, D., Arunachalam, R. M. and Aarthi, R. Distance Transform based Hand Gestures Recognition for Power point presentation Navigation. An Int. J. of Adv. Computing. 3(3) (May 2012), 41- 48 
[21] Gurjal, P. and Kunnur, K. Real time Hand Gesture Recognition using SIFT. Int. J. of Electronics \& Electrical Engg. 2(3) (March 2012), 19 - 33

[22] Rautaray, S. S. and Agarwal, A. Real time Hand Gesture Recognition System for Dynamic Applications. Int. J. of UbiComp, 3(1) (January 2012), 21 - 31

[23] Sultana, A. and Rajapuspha, T. Vision based Gesture Recognition for Alphabetical Hand Gestures using the SVM Classifier. Int. J. of Comp. Sc. And Engg. Tech. 3(7) (July 2012), $218-223$

[24] Ghotkar, A. S. and Kharate, G. K. Hand Segmentation Techniques to Hand Gesture Recognition for Natural Human Computer Interaction. Int. J. of Human Computer Interaction, 3(1) (2012) 15 - 25

[25] Kristensson, P. O., Nicholson, T. F. W. and Quigley, A. 2012. Continuous Recognition of One-handed and Twohanded Gestures using 3-D Full-body motion tracking sensors. In Proc. of IUI 12

[26] Rautaray, S. S. Real time Multiple Hand Gesture Recognition System for Human Computer Interaction. Int. J. of Intelligent Systems and Applications, 5 (May 2012), 56 - 64

[27] Kishore, P. V. V. and Kumar, P. R. Segment, Track, Extract, Recognize and Convert Sign Language Videos to Voice/ Text. Int. J. of Advance Comp. Sc. and Applications, 3(6) (2012) 35 - 47

[28] Trindade, P., Lobo, J. and BaHrreto, J. P. 2012. Hand Gesture Recognition using Color and Depth Images Enhanced with Hand Angular Pose Data. In Proc. of IEEE Int. Conf. on Multisensor Fusion \& Integration for Intelligent Systems

[29] Singh, S., Jain, A. and Kumar, D. Recognizing and Interpreting Sign Language Gesture for Human Robot Interaction. Int. J. of Computer Applications, 52 (11) (August 2012) 24 - 30

[30] Lahamy, H. and Lichti, D. 2012. Robust Real-Time and Rotation Invariant American Sign Language Alphabet Recognition using Range Camera. In Proc. of XXII ISPRS Congress

[31] Kishore, P. V. V. and Kumar, P. R. A Model for Real Time Sign Lang Recognition System. Int. J. of Adv. Research in Comp. Sc. And Software Engg. 2 (6) (June 2012), $29-35$

[32] Sharma, N. and Sharma, H. HIM: Hand Gesture Recognition in Mobile-learning. Int. J. of Comp. Applications, 44(16) (April 2012), 33 - 37

[33] Ghotkar, A. S., Khatal, R., Khupase, S., Asati, S. and Hadap, M. 2012. Hand Gesture Recognition for Indian Sing Language. In Proc. of Int. Conf. on Computer Communication and Informatics

[34] Bui, T. T. T., Phan, N. H. and Spitsyn, V. G. 2012. Face and Hand Gesture Recognition Algorithm Based on Wavelet transforms and Principal Component Analysis. In Proc. of 7th Int. Conf. on Strategic Technology.

[35] Kohn, B., Belbachir, A. N. and Nowakowska, A. 2012. Real-time Gesture Recognition using bio inspired 3D Vision Sensor. In Proc. of IEEE Comp. Society Conference on Comp. Vision and Pattern Recognition Workshops
[36] Kishore, P. V. V. and Kumar, P. R. A Video Based Indian Sign Language Recognition System (INSLR) Using Wavelet Transform and Fuzzy Logic. Int. J. of Engg. and Tech. 4(5) (October 2012), 537 - 542

[37] Geetha, M. and Manjusha, U. C. A Vision Based Recognition of Indian Sign Language Alphabets and Numerals Using B-Spline Approximation. Int. J. On Comp. Sc. and Engg. 4(3) (March 2012), 406 - 415

[38] Kurakin, A., Zhang, Z. and Liu, Z. 2012. A Real Time System for Dynamic Hand Gesture Recognition with a Depth Sensor. In Proc. Of 20th European Signal Processing Conference.

[39] Khan, R. Z. and Ibraheem, N. A. Hand Gesture Recognition: A Literature Review. Int. J. of Artificial Intelligence and Applications, 3(4) (July 2012), 161-173

[40] Suarez, J. and Murphy, R. R. 2012. Hand Gesture Recognition with Depth Images: A Review. In Proc. of Int. Sym. on Robot and Human Interactive Communication

[41] Kasprzak, W., Wilkowski, A. and Czapnik, K. Hand Gesture Recognition based on Free-Form Contours and Probabilistic Inference. Int. J. of Applied Math. and Comp. Sc. 22(2) (2012), 437- 448

[42] Jalab, H. A., Static Hand Gesture Recognition for Human Computer Interaction. Information Technology J. 11(09) (2012), 1265 - 1271

[43] Gowtham, P. N. V. S. A Hand Gesture Recognition based Virtual Touch World. Int. J. of Information \& Education Technology, 2(1) (Feb 2012), 36 - 4

[44] Shen, X., Hua, G., Williams, L. and Wu, Y. Dynamic Hand Gesture Recognition: An exemplar-based approach from motion divergence fields. J. of Image and Vision Computing, 30(3) (March 2012), 227 - 235

[45] Panwar, M. 2012. Hand Gesture Recognition based on Shape Parameters. In Proc. of Int. Conf. on Computing, Communication and Applications

[46] Pradhan, A., Ghose, M. K. and Pradhan, M. Hand Gesture Recognition using Feature Extraction. Int. J. of Current Engg. And Tech. 2(4) (Dec. 2012), 323 - 327

[47] Caputo, M., Denker, K., Dums, B. and Umlauf, G. 2012. 3D Hand Gesture Recognition based on Sensor Fusion of Commodity Hardware. In Proc. of Mensch \& Computer

[48] Dardas, N. H. and Georganas, N. D. Real-time Hand Gesture Detection and Recognition using Bag-ofFeatures and Support Vector Machine Techniques. IEEE Trans. on Instrumentation and Measurement, 60(11) (Nov 2011), $3592-3607$

[49] Panwar, M. and Mehra, P. S. 2011. Hand Gesture Recognition for Human Computer Interaction. In Proc. of Int. Conf. on Image Information Processing

[50] Ren, Z., Yuan, J and Zhang, Z. 2011. Robust Hand Gesture Recognition Based on Finger-Earth Mover's Distance with Commodity Depth Camera. In Proc. of the 19th ACM Int. Conf. on Multimedia

[51] Meena, S. 2011. A Study on Hand Gesture Recognition Technique. M. Tech. thesis. NIT, Rourkela (India)

[52] Bhuyan, M. K., Kar, M. K. and Neog, D. R. 2011. Hand Pose Identification from Monocular Image for Sign 
Language Recognition. In Proc. of IEEE Int. Conf. on Signal and Image Processing Applications

[53] Kumarage, D., Fernando, S., Fernando, P., Madushanka, D. and Samarasinghe, R. 2011. Real-time Sign Language Gesture Recognition Using Still-Image Comparison \& Motion Recognition. In Proc. of 6th Int. Conf. on Industrial and Information Systems.

[54] Dardas, N. H. and Petriu, E. M. 2011. Hand Gesture Detection and Recognition Using Principal Component Analysis. In Proc. of IEEE Int. conf on Computational Intelligence for Measurement Systems and Applications

[55] Guo, J. 2011. Hand Gesture Recognition and Interaction with 3D stereo Camera. Project Report. Australian National University

[56] Alsheakhali, M., Skaik, A, Aldahdouh, M. and Alhelou, M. 2011. Hand Gesture Recognition System. In Proc. of Int. Conf. on Information \& Communication Systems

[57] Ayala- Ramirez, V., Mota-Gutierrez, S. A., HernandezBelmonte, U. H. andd Sanchez-Yanez, R. E. 2011. A Hand Gesture Recognition System Based on Geometric Features and Color Information for Human Computer Interaction Tasks. In Proc. of Robotics Summer Meeting

[58] Rekha, J., Bhattacharya, J. and Majumder, S. 2011. Hand Gesture Recognition for Sign Language: A New Hybrid Approach. In Proc. of 15th International Conference on Image Processing, Computer Vision, \& Pattern Recognition

[59] Rekha, J., Bhattacharya, J. and Majumder, S. 2011. Shape, Texture and Local Movement Hand Gesture Features for Indian Sign Language Recognition. In Proc. of IEEE Int. Conf. on Trends in Information Sc. and Computing

[60] Priyal, S. P. and Bora, P. K. 2010. A study on Static Hand Gesture Recognition using Moments. In Proc. of Int. Conf. on Signal Processing and Communications

[61] Dardas, N. H. and Georganas, N. D. 2010. Hand Gesture Recognition Using Bag-of-Features and Multi-Class Support Vector Machine. In Proc. of IEEE Int. Sym. on Haptic Audio-Visual Environments and Games

[62] Elmezain, M., Al-Hamadi, A., Sadek, S. and Michaelis, B. 2010. Robust Method for Hand Gesture Spotting and Recognition Using Hidden Markov Model and Conditional Random Fields. In Proc. of IEEE Int. Symposium on Signal Processing and Information Tech.

[63] Yu, C., Wang, X., Huang, H., Shen, J. and Wu, K. 2010. Vision-Based Hand Gesture Recognition Using Combinational Features. In Proc. of 6th Int. Conf. on Intelligent Information Hiding and Multimedia Signal Processing

[64] Ravikiran, J., Mahesh, K., Mahishi, S., R., Dheeraj, S., Sudheender, Pujari, N. V. 2009. Finger Detection for Sign Language Recognition. In Proc. of Int. Multi Conf. of Engineers and Computer Scientists (Vol. 1)

[65] Elmezain, M., Al-Hamadi, A., Appenrodt, J. and Michaelis, B. 2008. A hidden Markov Model-Based Continuous Gesture Recognition System for Hand Motion Trajectory. In Proc. of 19th Int. Conf. on Pattern Recognition
[66] Paulraj, M.P., Yaacob, S., Desa, H., Hema, C. R., Ridzuan, W. M. and Majid, W. A. 2008. Extraction of Head and Hand Gesture Features for Recognition of Sign Language. In Proc. of Int. Conf. on Electronic Design

[67] Elmezain, M., Al-Hamadi, A., Krell, G., El-Etriby, S. and Michaelis, B. 2007. Gesture Recognition for Alphabets from Hand Motion Trajectory using Hidden Markov Models. In Proc. of IEEE Int. Symp. on Signal Processing and Information Tech.

[68] Fang, Y., Cheng, J., Wang, K. and Lu, H. 2007. Hand Gesture Recognition using First Multi-scale Analysis. In Proc. of 4th Int. Conf. on Image and Graphics

[69] Binh, N. D. and Ejima, T. 2006. Real-time Hand Gesture Recognition using Pseudo 3-D Hidden Markov Model. In Proc. of 5th IEEE Int. Conf. on Cognitive Informatics

[70] Vatavu, R. D., Pentiuc, S., Chaillou, C., Grisoni, L. and Degrande, S. 2006. Visual Recognition of Hand Postures for Interacting with Virtual Environment. In Proc. of Int Conf. on Development and Application Systems

[71] Chu, C. and Cohen, I. 2005. Posture and Gesture Recognition using 3D Body Shapes Decomposition. In Proc. of IEEE Computer Society Conf. on Computer Vision and Pattern Recognition

[72] Patwardhen, K. S. and Dutta Roy, S. 2004. Dynamic Hand Gesture Recognition using Predictive Eigen Tracker. In Proc. IUPRAI-sponsored Indian Conf. on Computer Vision, Graphics and Image Processing

[73] Chen, F., Fu, C. and Huang, C. Hand Gesture Recognition Using a Real-Time Tracking Method and Hidden Markov Models. J. of Image and Vision Computing. 21(8) (August 2003), $745-758$

[74] Wysoski, S. G., 2003. A Rotation Invariant Static Hand Gesture Recognition System using Boundary Information and Neural Networks. ME thesis. Nagoya Institute of Technology, Japan

[75] Sanchez-Nielsen, E., Anton-Canalís, L. and HernandezTejera, M. Hand Gesture Recognition for HumanMachine Interaction. J. of WSCG, 12 (1-3) (February 2003)

[76] Bretzner, L., Laptev, I. and Lindebergs, T. 2002. Hand Gesture Recognition using Multi-Scale Colour Features, Hierarchical Models and Particle Filtering. In Proc. of 5th IEEE Int. Conf. on Automatic Face and Gesture Recognition

[77] Jeong, M. H., Kuno, Y. and Shimada, N. 2002. Two Hand Gesture Recognition using Coupled Switching Linear Model. In Proc. of 16th IEEE Int. Conf. on Pattern Recognition (Vol. 1)

[78] Kim, J., Park, K., Bang, W. and Bien, Z. Z. 2002. Continuous Gesture Recognition System for Korean Sign Language based on Fuzzy Logic and Hidden Markov Model. In Proc. of IEEE Int. Conf on Fuzzy Systems

[79] Wysoski, S. G., Lamar, M. V., Kuroyanagi, S. and Iwata, A. 2002. A Rotation Invariant Approach on StaticGesture Recognition using Boundary Histograms \& Neural Networks. In Proc. of the 9th Int. Conf. on Neural Information Processing, (Vol.4)

[80] MacLean, J., Herpers, R., Pantofaru, Wood, C., L., Derpanis, K., Topalovic, D. and Tsotsos, J. 2001. Fast 
Hand Gesture Recognition for Real-Time Teleconferencing Applications. In Proc. of IEEE ICCV Workshop on Recognition, Analysis, and Tracking of Faces and Gestures in Real-Time Systems

[81] Yoon, H., Soh, J., Bae, Y. J. and Yang, H. S. Hand Gesture Recognition using Combined Features of Location, Angle and Velocity. J. of Pattern Recognition Society, 34 (2001)

[82] Jeong, M. H., Kuno, Y. and Shimada, N. 2001. Recognition of Shape-Changing Hand Gestures Based on Switching Linear Model. 11th Int. Conf. on Image Analysis and Processing

[83] Lamar, M.V., Bhuiyan, Md. S. and Iwata, A. 1999. Hand Gesture Recognition using Morphological Principal Component Analysis and an Improved CombNET-II. In Proc. IEEE Int. Conf. on Systems, Man, and Cybernetics (Vol. 4)

[84] Liang, R. and Ouhyoung, M. 1998. A Real-time Continuous Gesture Recognition System for Sign Language. In Proc. 3rd IEEE Int. Conf. on Automatic Face and Gesture Recognition

[85] Min, B., Yoon, H., Soh, J., Yang, Y. and Ejima, T. 1997. Hand Gesture Recognition using Hidden Markov Models. In Proc. of IEEE Int. Conf. on Systems, Man, \& Cybernetics (Vol. 5)

[86] Tsukamoto, A. and Lee, C. 1995. A Methodological Approach on Real-time Gesture Recognition using Multiple Silhouette Models. In Proc. of 4th IEEE Int. Workshop on Robot and Human Communication
[87] Ishibuchi, K., Takemura, H. and Kishino, F. 1993. Real Time Hand Gesture Recognition using 3D Prediction Model. In Proc. of Int. Conf. on Systems, Man and Cybernetics (Vol.5)

[88] Tamersoy, B. 2009. Background Subtraction - Lecture Notes. University of Texas at Austin.

[89] Ziou, D. and Tabbone, S. 1998. Edge Detection Techniques - An Overview. Int. J. of Pattern Recognition and Image Analysis, Vol. 8, pp. 537 -559

[90] Maini, R. and Aggarwal, H. Study and Comparison of Various Image Edge Detection Techniques. Int. J. of Image Processing, 3(1)

[91] Lin, R. S. 2008. Edge Detection by Morphological Operations and Fuzzy Reasoning. In Proc. of Congress on Image and Signal Processing, May 2008, China, pp. $729-733$

[92] Gorodnichy, D. O. and Yogeswaran, A. (2006). Detection and Tracking of Pianist Hands and Fingers. In Proc. of 3rd Canadian Conference on Computer and Robot Vision

[93] Nölker, C. and Ritter, H., (1997). Detection of Fingertips in Human Hand Movement Sequences. In Proc. of International Gesture Workshop

[94] E. Stergiopoulou, N. Papamarkos. Hand gesture recognition using a neural network shape fitting technique. J. of Engineering Applications of Artificial Intelligence, 22(8), (2009) 